\title{
Baryonic contributions to the dilepton spectrum of nucleon-nucleon collisions
}

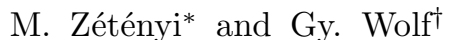 \\ KFKI Research Institute for Nuclear and Particle Physics, H-1525 Budapest 114, POB. 49, Hungary
}

(Dated: November 20, 2018)

\begin{abstract}
We study the production of dileptons in relativistic nucleon-nucleon collisions. Additionally to the traditional dilepton production channels (vector meson decays, meson and $\Delta(1232)$ Dalitz decays) we included in our model as new dilepton sources the Dalitz decay of higher unflavored baryon resonances with $\operatorname{spin} \leq 5 / 2$ and mass $\leq 2.25 \mathrm{GeV} / \mathrm{c}^{2}$. The contributions of these new channels are estimated using experimental information about the $N \gamma$ decays of the resonances and have large uncertainties. The obtained dilepton spectra are compared to the experimental data by the DLS collaboration. Predictions for the HADES detector (SIS, GSI) are also discussed. In spite of the large uncertainties of the higher resonance Dalitz decay contributions we are able to draw the conclusion that these contributions are negligible compared to the other dilepton sources and do not influence the detectability of the $\phi$ and $\omega$ vector meson peaks.
\end{abstract}

PACS numbers: 13.75.Cs; 13.30.Ce; 13.40.Hq; 14.20.Gk

\section{INTRODUCTION}

The main objective of the physics program at SIS (GSI, Darmstadt) is the study of hot and dense hadronic matter formed in nucleus-nucleus collisions. The clearest probes for this investigation are dileptons, which leave the interaction volume unaffected by strong final state interactions. The purpose of the HADES detector is to study the dilepton spectrum produced in nucleus-nucleus, nucleon-nucleus, nucleon-nucleon, and pion-nucleus collisions.

Theoretical investigations predict a partial restoration of chiral symmetry in hot and dense nuclear matter, which results in a modification of particle properties. For an experimental study of these phenomena the light vector mesons $\rho, \omega$, and $\phi$ seem to be a suitable probe. The $\rho$-owing to its short lifetime - decays inside the hot and dense hadronic matter. Predicted in-medium modifications of $\omega$ and $\phi$ may result in a considerable growth of their decay widths and there is hope that they also decay inside the hot and dense phase. On the other hand, $\rho, \omega$, and $\phi$ decay directly to dileptons, which allows a clear experimental study.

Dileptonic decays of vector mesons contribute to the dilepton invariant mass spectrum as peaks or - in the case of the $\rho$ - as a wider distribution. In-medium modification of vector meson masses and decay widths may be identified in the dilepton spectrum as a shift and widening of the corresponding peaks, respectively.

To identify the vector mesons in the dilepton spectrum a careful analysis of the backgrounds is needed. One of the possible sources of background is the Dalitz-decay of baryon resonances, $N^{*} \rightarrow N e^{+} e^{-}$. These processes may be interesting also for themselves since baryon resonance properties may also be subject to in-medium modifications. In earlier studies only the $\Delta(1232)$ and $N(1440)$ Dalitz-decays have been considered in model calculations (the latter giving a negligible contribution). On the other hand, some of the higher resonances have a relatively high photonic decay width and are, therefore, expected to have a non-negligible contribution to the dilepton spectrum through their Dalitz-decay. The kinematical upper limit for the mass of dileptons resulting from the Dalitz-decay of resonances rises with increasing resonance mass, therefore higher mass resonances may contribute to the dilepton spectrum in a higher dilepton mass range where the contribution of the $\Delta(1232)$ is already negligible. It is particularly interesting to see if some of the higher mass resonances contribute essentially to the dilepton invariant mass spectrum in the $\phi$ mass region since this may influence the possible identification of the $\phi$ meson in the dilepton spectrum. Some of the higher mass resonances have also higher spins. In studying their electromagnetic transitions special care should be taken of their higher spin nature.

Dilepton production in proton-proton collisions with beam energies between 1 and $5 \mathrm{GeV}$ has been studied experimentally by the DLS collaboration [1], supplying an opportunity to test models of dilepton production in elementary processes. These models may in turn be used as an input in transport models of heavy ion collisions.

In this paper we discuss the Dalitz-decay of baryon resonances up to spin-5/2. We inserted our results into a Monte Carlo model of relativistic proton-proton collisions and obtained the dilepton invariant mass spectra.

*Electronic address: zetenyi@rmki.kfki.hu

${ }^{\dagger}$ Electronic address: wolf@rmki.kfki.hu 
The lack of experimental information on the Dalitz-decay of baryon resonances forces one to use information on the - often also poorly known - radiative decays, $N^{*} \rightarrow N \gamma$. This brings in several assumptions and leads to uncertainties of the results. The resulting uncertainty of the total dilepton invariant mass spectrum is moderate and - as we will see - allows one to draw the conclusion that the detectability of the $\phi$ and $\omega$ meson peaks is not influenced by the baryon resonance Dalitz-decays.

In an earlier paper [2] we discussed the calculation of the Dalitz-decay of baryon resonances and made a comparison of the contributions of the various possible decay amplitudes. In Sec. I of the present paper we briefly review those results. In Sec. III we give a short description of our Monte Carlo model for proton-proton collisions, in Sec. IIIB we discuss the dilepton production channels included in the model. In Secs. IIIC and IIID we compare our dilepton spectra with the DLS data and give predictions for the HADES detector. Throughout the paper we set $c=\hbar=1$.

\section{DALITZ-DECAY OF A SPIN- $J$ RESONANCE}

\section{A. Kinematics and matrix elements}

Let $p^{*}, m^{*}$, and $\lambda^{*}$ denote the four-momentum, mass, and helicity of the decaying resonance, respectively, and $p, m$, and $\lambda$ the corresponding quantities for the nucleon. Let us introduce the notation $q=p^{*}-p$ for the photon four-momentum, $M^{2}=q^{2}$ for the virtual photon mass (= dilepton invariant mass) squared, and $P=\left(p^{*}+p\right) / 2$.

The differential width of the Dalitz-decay of a particle is related to its photonic decay width to a virtual photon $\Gamma_{N^{*} \rightarrow N \gamma}(M)$ by [3]

$$
\frac{d \Gamma_{N^{*} \rightarrow N e^{+} e^{-}}}{d M^{2}}=\frac{\alpha}{3 \pi} \frac{1}{M^{2}} \Gamma_{N^{*} \rightarrow N \gamma}(M)
$$

Here $\Gamma_{N^{*} \rightarrow N \gamma}(M)$ can be expressed in terms of the photonic decay matrix element $\left\langle N \gamma|T| N^{*}\right\rangle$ as

$$
\Gamma_{N^{*} \rightarrow N \gamma}(M)=\frac{\sqrt{\lambda\left(m^{* 2}, m^{2}, M^{2}\right)}}{16 \pi m^{* 3}} \frac{1}{n_{p o l, N^{*}}} \sum_{p o l}\left|\left\langle N \gamma|T| N^{*}\right\rangle\right|^{2},
$$

where

$$
\left\langle N \gamma|T| N^{*}\right\rangle=-\epsilon^{\mu}\left\langle N\left|J_{\mu}\right| N^{*}\right\rangle
$$

with $\epsilon^{\mu}$ the photon polarization vector, and $J_{\mu}$ the electromagnetic current operator. In (2) $n_{\text {pol, } N^{*}}$ is the number of polarization states of the $N^{*}$ resonance and $\lambda(a, b, c)=a^{2}+b^{2}+c^{2}-2(a b+b c+a c)$ is the usual kinematical factor.

Next we determine the matrix element $\left\langle N\left|J_{\mu}\right| N^{*}\right\rangle$ for an $N^{*}$ resonance of spin- $J$. A spin- $J(J \geq 3 / 2)$ fermion can be described by a spinor-tensor field $\Psi^{\rho_{1} \cdots \rho_{n}}(n=J-1 / 2)$, therefore in momentum-space matrix elements the particle is represented by a spinor-tensor amplitude $u^{\rho_{1} \cdots \rho_{n}}\left(p^{*}, \lambda^{*}\right)$, which has to fulfill the generalized Rarita-Schwinger relations in order to eliminate the different spin degrees of freedom. The electromagnetic current matrix element can be written generally as

$$
\left\langle N\left|J_{\mu}\right| N^{*}\right\rangle=\bar{u}(p, \lambda) \Gamma_{\mu \rho_{1} \cdots \rho_{n}} u^{\rho_{1} \cdots \rho_{n}}\left(p^{*}, \lambda^{*}\right),
$$

where the form of $\Gamma_{\mu \rho_{1} \cdots \rho_{n}}$ is restricted by the conservation of electric charge, $q^{\mu}\left\langle N\left|J_{\mu}\right| N^{*}\right\rangle=0$. As a result of the Dirac equations $\bar{u}(p, \lambda)(\not p-m)=0$ and $\left(\not p^{*}-m^{*}\right) u^{\rho_{1} \cdots \rho_{n}}\left(p^{*}, \lambda^{*}\right)=0$ and the generalized Rarita-Schwinger relations $\Gamma_{\mu \rho_{1} \cdots \rho_{n}}$ contains only three independent terms:

$$
\Gamma_{\mu \rho_{1} \cdots \rho_{n}}=\sum_{i=1}^{3} f_{i}\left(M^{2}\right) \chi_{\mu \rho_{1}}^{i} p_{\rho_{2}} \cdots p_{\rho_{n}} G
$$

with

$$
\begin{aligned}
& \chi_{\mu \rho}^{1}=\gamma_{\mu} q_{\rho}-\not q g_{\mu \rho}, \\
& \chi_{\mu \rho}^{2}=P_{\mu} q_{\rho}-(P \cdot q) g_{\mu \rho}, \\
& \chi_{\mu \rho}^{3}=q_{\mu} q_{\rho}-M^{2} g_{\mu \rho},
\end{aligned}
$$

and $G=1$ or $\gamma_{5}$ for resonances with positive or negative normalities, respectively. The normality of a spin- $J$ baryon is by definition $P(-1)^{J-1 / 2}$ with $P$ the intrinsic parity. $f_{i}\left(M^{2}\right), i=1,2,3$ are three independent form factors, which should be determined from experimental data. 


\section{B. Parameter fitting}

In our model we neglect the $M^{2}$ dependence of the form factors, therefore $f_{i}$ become constant couplings. (Vector meson dominance does not apply here since we include the $\rho$ dileptonic decay separately.) We redefine the $\chi_{\mu \rho}^{i}$ in our matrix elements dividing them by the appropriate power of the nucleon mass $m$ in order to render the form factors $f_{i}$ dimensionless. We, further, write $f_{i}=e g_{i}$, with $e$ the elementary charge related to the fine structure constant by $e^{2}=4 \pi \alpha$. We will use the real photonic decay width of the resonances to determine the dimensionless coupling constants $g_{i}$.

A detailed investigation of the Dalitz-decay width calculated from the 3 independent matrix elements and the interference terms leads to the following conclusions (see [2] for details):

- Those terms of the Dalitz-decay width that contain $\chi_{\mu \rho}^{3}$ give zero contribution at $M=0$ (real photons), therefore information on the real photonic width of the resonances can not be used to fix the value of the $g_{3}$ coupling.

- The remaining terms $\left(\propto g_{1}^{2}, g_{2}^{2}\right.$ and $\left.g_{1} g_{2}\right)$ give very similar contributions to the Dalitz-decay width. Thus an arbitrary choice between the $\chi_{\mu \rho}^{1}$ and $\chi_{\mu \rho}^{2}$ terms causes only a minor uncertainty in the dilepton invariant mass spectrum, that is definitely smaller than the uncertainty arising from the poor knowledge of the photonic width in the case of most of the resonances.

- The resonance mass dependence of the Dalitz-decay width may influence the resulting dilepton spectrum because the masses of the resonances are generated according to a Breit-Wigner distribution in our model (see Sec. IIIA), but we use a single coupling fitted to the photonic width of a resonance with its mass equal to the peak value. However, the Breit-Wigner distribution ensures that resonance masses stay close to the peak value and hence the uncertainty of the dilepton spectrum caused by the different resonance mass dependence of the $\chi_{\mu \rho}^{1}$ and $\chi_{\mu \rho}^{2}$ is small.

These results encourage us to make the assumption that $g_{2}=g_{3}=0$, i.e., only $\chi_{\mu \rho}^{1}$ (the term containing the lowest power of external momenta) contributes to the Dalitz-decay of baryon resonances.

To demonstrate the relevance of the spin-parity of baryon resonances in their Dalitz-decays we show in Fig. 1 the differential Dalitz-decay width of hypothetical resonances with the same mass and photonic width but with different spin-parities. It can be seen from the plot that at the high dilepton mass end of the spectrum spin-parity $1 / 2+$ and $3 / 2$ - resonances give the largest while $5 / 2$ - give the smallest contribution to the dilepton spectrum.

\section{DILEPTON PRODUCTION IN PROTON-PROTON COLLISIONS}

\section{A. The model}

We use a resonance model to describe particle production in proton-proton collisions: baryon resonances are produced in the first step, which then decay - possibly in a multi-step process - into the final state particles. The resonances are treated as on-shell particles in their production processes, but their mass is generated according to a Breit-Wigner distribution. We included in our model all unflavored baryon resonances with mass $\leq 2.25 \mathrm{GeV}$, and with a status of at least 3 stars according to the Review of Particle Physics [4], i.e., $\Delta(1232), N(1440), N(1520), N(1535)$, $\Delta(1600), \Delta(1620), N(1650), N(1675), N(1680), N(1700), \Delta(1700), N(1710), N(1720), \Delta(1905), \Delta(1910), \Delta(1920)$, $\Delta(1930), \Delta(1950), N(2190), N(2220), N(2250)$, and some additional resonances with weaker status, namely $\Delta(1900)$, $N(2000)$, and $N(2080)$. In our model the following decay channels of baryon resonances are taken into account: $N \pi$, $N \eta, N \pi \pi$ (including $N \rho, \Delta(1232) \pi, N(1440) \pi$, and $N " \sigma "), N \omega, \Sigma K$, and $\Lambda K$.

We fitted the resonance properties (branching ratios, full widths, and masses within the errors given in the Review of Particle Physics [4]) to experimental data of pion induced reactions. Cross sections of resonance production have been fitted to one-pion, two-pion, $\eta, \omega, \rho$, and kaon production in proton-proton and proton-neutron collisions. Formulae for resonance and meson production cross sections used in the fits are essentially the same as in the BUU model established by Gy. Wolf (see [5] and references therein). We needed to include in the model the resonances with weaker status mentioned above in order to obtain a good fit. For further details of parameter fitting see [6].

\section{B. Dilepton production}

We used the above model to calculate the dilepton invariant mass spectrum of proton-proton collisions. The dilepton sources considered are the direct dilepton decay of $\rho^{0}, \omega$, and $\phi$ vector mesons and Dalitz-decay of $\pi^{0}, \eta$, and $\omega$ mesons, and baryon resonances. For the meson Dalitz-decay channels we used the same expressions as in [5]. 
The differential cross section of dilepton production in the direct decay of a $V$ vector meson is given by

$$
\frac{d \sigma(s, M)}{d M}^{p p \rightarrow p p V \rightarrow p p e^{+} e^{-}}=\frac{d \sigma(s, M)}{d M}^{p p \rightarrow p p V} B R_{V \rightarrow e^{+} e^{-}}(M) .
$$

Here $B R_{V \rightarrow e^{+} e^{-}}(M)$ is the dileptonic branching ratio of the vector meson $V$, which is approximated in the case of the $\rho$ as

$$
B R_{\rho \rightarrow e^{+} e^{-}}(M)=\frac{\Gamma_{\rho \rightarrow e^{+} e^{-}}(M)}{\Gamma_{\rho \rightarrow \pi \pi}(M)+\Gamma_{\rho \rightarrow e^{+} e^{-}}(M)+\Gamma_{\rho \rightarrow \pi \gamma}(M)},
$$

where we adopt the mass dependence of the pionic and dileptonic $\rho$ width from [7] while we keep $\Gamma_{\rho \rightarrow \pi \gamma}$ constant, determining its value from the Breit-Wigner with and branching ratio listed in the Review of Particle Physics [4]. The appearance of $\Gamma_{\rho \rightarrow e^{+} e^{-}}$and $\Gamma_{\rho \rightarrow \pi \gamma}$ in the denominator of (8) is only relevant close to the two pion threshold $M=2 m_{\pi}$, where $\Gamma_{\rho \rightarrow \pi \pi}$ becomes small.

We keep the dileptonic branching ratio of the narrow $\omega$ and $\phi$ resonances constant and use their PDG values. $\phi$ meson production is not handled by our resonance model and the production cross section is not well known experimentally. This leads us to adopt here the $\phi$ meson production cross section calculated from a one-boson exchange model [8].

We calculate the dilepton spectra from the Dalitz-decay of those of the above mentioned baryon resonances that have spin $\leq 5 / 2$ and are known to couple to the $N \gamma$ channel according to the PDG, i.e., $\Delta(1232), N(1440), N(1520)$, $N(1535), \Delta(1600), \Delta(1620), N(1650), N(1675), N(1680), N(1700), \Delta(1700), N(1710), N(1720), \Delta(1905), \Delta(1910)$, $\Delta(1930)$. For resonances with spin $\geq 3 / 2$ we use the results of the previous section. The Dalitz-decay of the $N(1440)$ resonance has already been included in Ref. [9]. We treated all the other spin- $1 / 2$ resonances in the same fashion.

To fix the electromagnetic coupling constants $g_{1}$ defined in Sec. I the photonic width of the resonances is needed. This we calculate using the full width of the resonances (as obtained in our resonance model) and the photonic branching ratios, that we take from the Review of Particle Physics [4]. We made calculations using both the minimal and the maximal values of the photonic branching ratios given in [4]. This provides us with an estimate of the resulting uncertainty of the dilepton production cross sections.

Carrying out the parameter fitting described in Sec. [II we arrived at two different parameter sets describing the experimental data with nearly the same accuracy. We calculated the dilepton spectrum with both parameter sets. From the point of view of dilepton production the main difference between the two parameter sets is in the baryon resonance Dalitz-decay channels: using the parameters of our best fit two of the higher resonance channels, the $N(1520)$ and $N(1535)$ give considerable contributions to the dilepton spectrum. The contribution of these two channels exceeds that of the $\Delta(1232)$ at the large dilepton mass end of the spectrum for large enough beam energies. In the case of the other parameter set the $N(1520)$ gives a smaller contribution and its role is partially taken over by the $\Delta(1700)$, which gives a larger contribution for large dilepton masses. We used the parameters of our best fit in obtaining all numerical results presented in the rest of the paper.

\section{Comparison with the DLS data}

In Fig. 22 we plot the dilepton invariant mass spectra $d \sigma / d M$ for $p p$ collisions calculated from our model in comparison to the DLS data [1]. We have taken into account the DLS experimental filter and the finite dilepton mass resolution of the detector. In the plots contributions of the following sources are shown: direct dileptonic decay of $\rho^{0}, \omega$, and $\phi$ vector mesons, Dalitz-decay of $\pi^{0}, \eta$, and $\omega$ mesons, and Dalitz-decay of $\Delta(1232), N(1520)$ and $N(1535)$ baryon resonances. The total cross section is a sum of all sources including the Dalitz-decay of the other baryon resonances.

A first glance at Fig. 2 shows that although the resonance Dalitz-decay channels, esp. the $N(1535)$ channel has a large uncertainty arising from the poor knowledge of the photonic branching ratios, the resulting relative uncertainty of the full dilepton production cross section is not dramatic.

The dominant dilepton sources at the two lowest beam energies are the $\pi^{0}$ and $\Delta(1232)$ Dalitz-decay, and direct dilepton decay of $\rho^{0}$. At $1.04 \mathrm{GeV}$ the experimental data are underestimated by the model. Comparison with results of other models successfully describing the data [7, 10, 11] suggests that the reason is probably the underestimation of the $\Delta(1232)$ channel at this energy.

For beam energies $E_{b} \geq 1.61 \mathrm{GeV}$ the $\eta$ channel gives the dominant contribution around $0.2 \mathrm{GeV}$ dilepton mass. Figure 3 shows a comparison of the $\eta$ meson production cross section deduced from our model to the available experimental data [12, 13]. Our model underestimates the two data points at $E_{b}=2.02 \mathrm{GeV}\left(p_{l a b}=2.81 \mathrm{GeV}\right)$. As a result we also underestimate the dilepton cross section in the $M=0.2-0.4 \mathrm{GeV}$ range for the beam energies $E_{b}=$ $1.85 \mathrm{GeV}$ and $2.09 \mathrm{GeV}$ (and, to a lesser extent, for $E_{b}=1.61 \mathrm{GeV}$ ). 
The peak appearing in the dilepton spectrum of the $\rho$ channel at $M \sim 0.4 \mathrm{GeV}$ is a result of two reasons. First, low mass $\rho$ mesons are produced with a high cross section via the decay of the $N(1520)$ resonance, therefore also the $\rho$ mass spectrum has a peak in the same region. Second, close to the two pion threshold the width of the decay $\rho \rightarrow \pi \pi$ becomes small, consequently the dileptonic branching ratio $B R_{\rho \rightarrow e^{+} e^{-}}(M)$, that enters the expression of the dileptonic differential cross section (7), rises rapidly as $M$ approaches $2 m_{\pi}$.

In the $M>0.4 \mathrm{GeV}$ region the experimental dilepton spectra are more or less well described by the model for the beam energies $1.27 \mathrm{GeV} \leq E_{b} \leq 2.09 \mathrm{GeV}$ with the exception of a discrepancy around $M=0.6 \mathrm{GeV}$ for $E_{b}=$ $2.09 \mathrm{GeV}$. The same underestimation of the data was seen using the model [10] and a strong $p p$ bremsstrahlung contribution was named as a possible explanation.

At $4.88 \mathrm{GeV}$ beam energy channels with additional pions in the final state are known to dominate the dilepton spectrum [ 10, 11]. These models use a string fragmentation model to describe the DLS data. Our model, since it does not handle final states with additional pions, looses its validity at this beam energy. Consequently, our results are an order of magnitude below the experimental data.

\section{Predictions for HADES}

Figure 4 shows the dilepton invariant mass spectra calculated from our model for the beam energies $E_{b}=3 \mathrm{GeV}$ and $3.5 \mathrm{GeV}$. Here no experimental filter has been used. In choosing the beam energies we have taken into account that here we are primarily interested in the Dalitz-decay channels of higher baryon resonances, which may have an important contribution in the higher dilepton mass region where the contribution of the $\Delta(1232)$ is already small, i.e., around $M=m_{\phi}$. Therefore, we have chosen beam energies above the $\phi$ meson production threshold of $E_{b}=2.59$ $\mathrm{GeV}$. On the other hand, one must not go beyond the beam energy where the string channel opens and the model looses its validity [10].

In Fig. 4 we see that the dominant dilepton sources at these energies are the $\pi^{0}$ and $\eta$ Dalitz-decay and the direct dilepton decays of vector mesons. Both the $N(1535)$ and the $N(1520)$ channels exceed the $\Delta(1232)$ contribution in the $\phi$ mass region, but they remain well below the $\phi$ meson contribution. Consequently, a pronounced peak appears at the $\phi$ mass in the total dileptonic differential cross section. A similar peak is seen at the $\omega$ mass. These peaks should be clearly identified by a high mass resolution dilepton spectrometer such as HADES. We note that we found the same qualitative features in the case of the second resonance parameter set, namely, the $\phi$ and $\omega$ peaks are clearly visible in spite of the fact that the $\Delta(1700)$ channel exceeds the $\Delta(1232)$ contribution in the $\phi$ mass region.

\section{SUMMARY}

In this paper we investigated the production of dileptons in relativistic proton-proton collisions using a Monte Carlo model. We introduced as new channels the Dalitz-decay of a number of higher baryon resonances. The lack of experimental information about the Dalitz decay of higher resonances causes a large uncertainty of the calculated dilepton spectra of these channels. We have given an estimate of the uncertainties arising from the poor knowledge of the $N \gamma$ branching ratios of the baryon resonances. Despite the large uncertainties we may conclude that the Dalitz decay of higher baryon resonances gives a negligible contribution to the dilepton spectrum. The dominant dilepton sources are the $\pi^{0}$ and $\eta$ Dalitz-decay and the direct dilepton decays of vector mesons.

The obtained dilepton spectra compare well with the experimental data of the DLS collaboration for beam energies $E_{b} \leq 2.09 \mathrm{GeV}$ with some exceptions. The most serious disagreement between our results and the experimental data can be explained by comparing the dilepton and the $\eta$ production data at the same energies. We have also given predictions for the HADES detector for beam energies above the $\phi$ meson production threshold. Our model predicts clear peaks at the $\omega$ and $\phi$ meson masses, which should be easily identified by the HADES detector.

Our model can also be used as an input to a transport model of heavy ion collisions, where in-medium modifications of vector mesons and baryon resonances can be studied. 


\section{Acknowledgments}

This work was supported by the National Fund for Scientific Research of Hungary, grant Nos. OTKA T30171, T30855, and T32038, and an MTA-DFG project.

[1] W. K. Wilson et al., Phys. Rev. C 57 (1998) 1865.

[2] M. Zétényi and Gy. Wolf, accepted for publication in Heavy Ion Phys, nucl-th/0202047

[3] B. E. Lautrop and J. Smith, Phys. Rev. D 3 (1971) 1122.

[4] K. Hagiwara et al., Phys. Rev. D 66, 010001 (2002) 1.

[5] Gy. Wolf, Heavy Ion Phys. 5 (1997) 281.

[6] Gy. Wolf, in preparation.

[7] E. L. Bratkovskaya, W. Cassing, M. Effenberger, and U. Mosel, Nucl. Phys. A653 (1999) 301.

[8] W. S. Chung, G. Q. Li, and C. M. Ko, Phys. Lett. B401 (1997) 1.

[9] Gy. Wolf, G. Batko, W. Cassing, U. Mosel, K. Niita, and M. Schäfer, Nucl. Phys. A517 (1990) 615.

[10] C. Ernst, S. A. Bass, M. Belkacem, H. Stöcker, and W. Greiner, Phys. Rev. C 58 (1998) 447.

[11] E. L. Bratkovskaya, W. Cassing, and U. Mosel, Nucl. Phys A686 (2001) 568.

[12] Baldini et al., Landolt-Börnstein vol. 12, (Springer, Berlin, 1987).

[13] E. Chiavassa, G. Dellacasa, N. De Marco, C. De Oliveira Martins, M. Gallio, P.Guaita, A. Musso, A. Piccotti, E. Scomparin, E. Vercellin, Phys. Lett. B322 (1994) 270. 


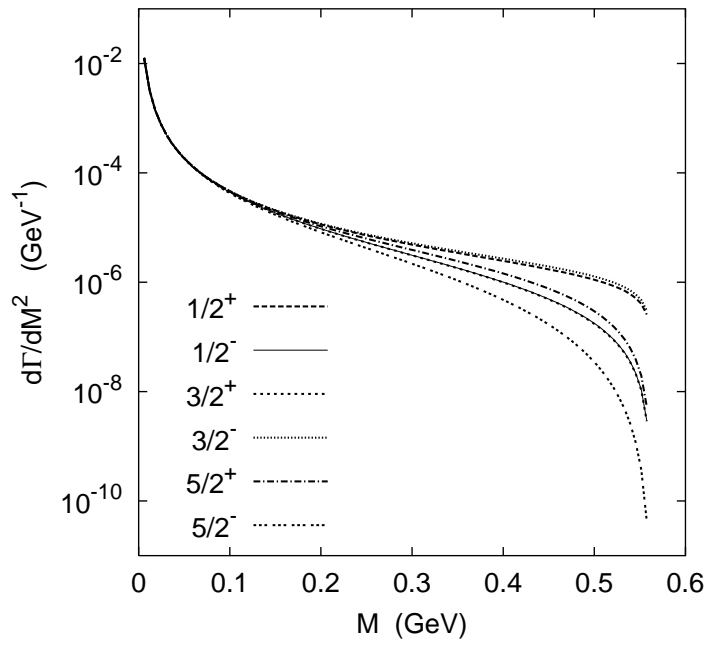

FIG. 1: Differential width of the Dalitz-decay of hypothetical baryon resonances with the same mass $(1.5 \mathrm{GeV})$ and photonic width $(0.6 \mathrm{MeV})$, but with different spin-parities. Curves for $1 / 2-$ and $3 / 2+$ resonances nearly overlap, similarly $1 / 2+$ and $3 / 2-$ curves. 

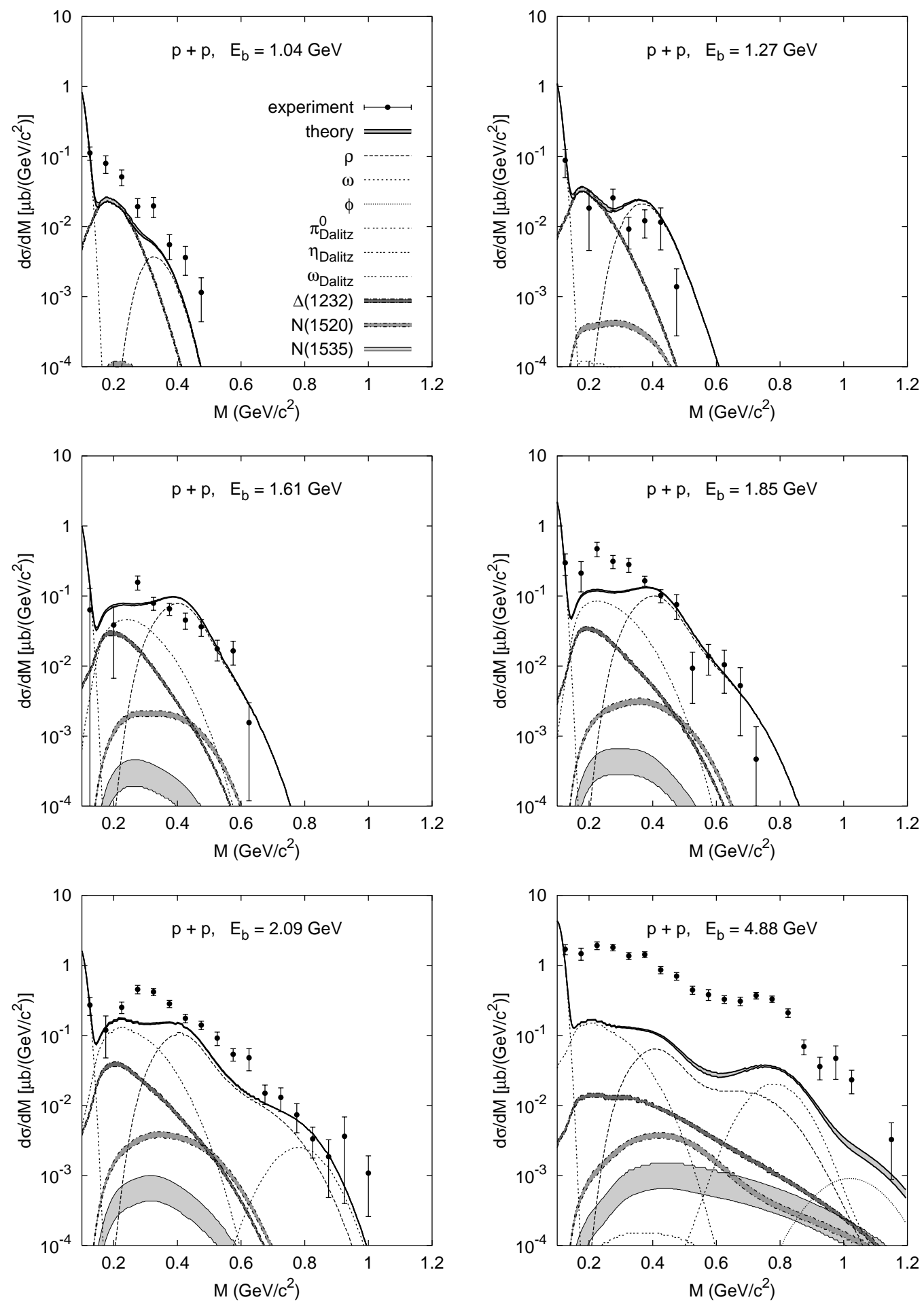

FIG. 2: The dilepton invariant mass spectra for $p p$ collisions calculated from our model taking into account the DLS filter and the finite mass resolution in comparison to the DLS data. For the meaning of the various line styles see the explanation in the plot for $1.04 \mathrm{GeV}$ beam energy. The solid line represents the sum of all channels including those that are not indicated in the plots (i.e. Dalitz-decay of the other baryon resonances). In the case of baryon resonance Dalitz-decays the lower (upper) edge of the shaded region corresponds to the dilepton production cross section calculated using the minimal (maximal) value of the photonic branching ratio given in the Review of Particle Physics. The resulting uncertainty of the total dilepton spectrum is indicated similarly. 


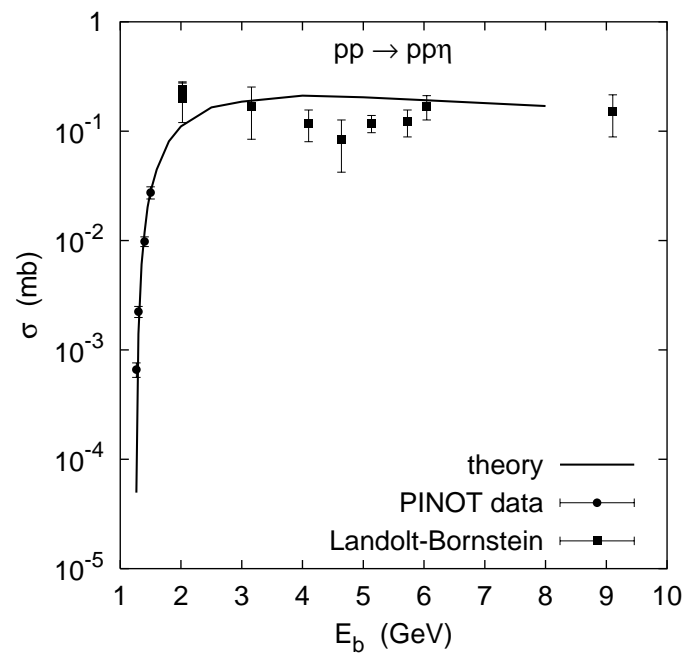

FIG. 3: Cross section of $\eta$ meson production as a function of the beam energy. The solid line represents the results from our model while full circles and squares show the experimental data.
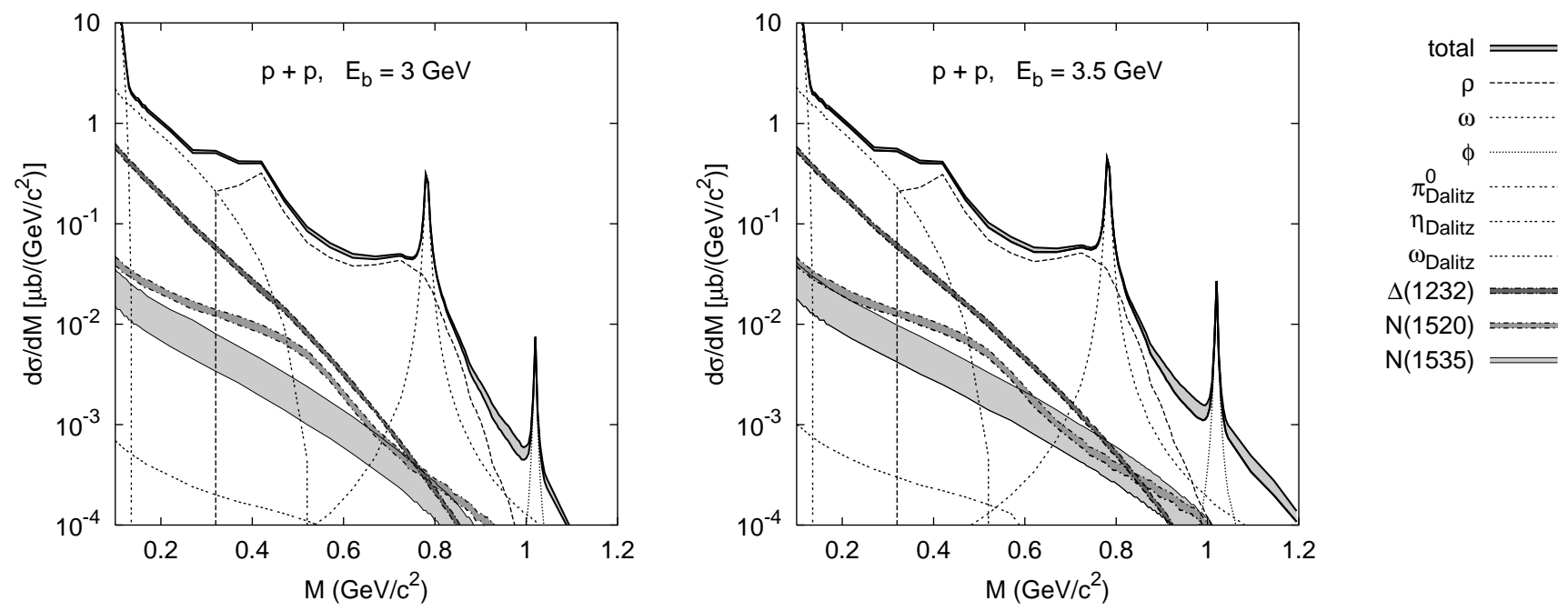

FIG. 4: Dilepton invariant mass spectrum from proton-proton collisions at various beam energies. No experimental filter has been used. The uncertainties of the baryon resonance Dalitz-decay channels and the total dilepton spectrum are indicated similarly to Fig. 2 